\section{Dopamine in amygdala gates limbic processing of aversive stimuli in humans}

\author{
Thorsten Kienast ${ }^{1}$, Ahmad R Hariri ${ }^{2}$, Florian Schlagenhauf ${ }^{1}$, \\ Jana Wrase ${ }^{1}$, Philipp Sterzer ${ }^{1}$, Hans Georg Buchholz ${ }^{3}$, \\ Michael N Smolka ${ }^{4}$, Gerhard Gründer ${ }^{5}$, Paul Cumming ${ }^{6}$, \\ Yoshitaka Kumakura ${ }^{7}$, Peter Bartenstein ${ }^{6}$, Raymond J Dolan ${ }^{8}$ \& \\ Andreas Heinz ${ }^{1}$ \\ Dopamine is released under stress and modulates processing \\ of aversive stimuli. We found that dopamine storage capacity \\ in human amygdala, measured with 6-[ $\left.{ }^{18} \mathrm{~F}\right]$ fluoro-L-DOPA \\ positron emission tomography, was positively correlated with \\ functional magnetic resonance imaging blood oxygen level- \\ dependent signal changes in amygdala and dorsal anterior \\ cingulate cortex that were evoked by aversive stimuli. \\ Furthermore, functional connectivity between these two regions \\ was inversely related to trait anxiety. Our results suggest that \\ individual dopamine storage capacity in amygdala subserves \\ modulation of emotional processing in amygdala and dorsal \\ cingulate, thereby contributing to individual differences in \\ anxious temperament.
}

An emphasis is frequently placed on the importance of dopamine in shaping complex behavior and in regulating neural responses to rewarding stimuli ${ }^{1}$, often overshadowing its contribution to aversive processing ${ }^{2-4}$. Animal studies demonstrate that dopamine release in the amygdala augments excitatory sensory input and attenuates inhibitory prefrontal input, resulting in augmented aversive conditioning ${ }^{5}$. Consistent with these findings, human pharmacological neuroimaging studies show that increased dopamine release potentiates human amygdala function ${ }^{6,7}$. These convergent findings suggest that variability in dopamine signaling may contribute to individual variability in amygdala function and related emotional behaviors.

Blood oxygen level-dependent (BOLD) functional magnetic resonance imaging (fMRI) is an effective and reliable tool for measuring stable individual differences in amygdala function ${ }^{8}$. Positron emission tomography (PET) recordings with $6-\left[{ }^{18} \mathrm{~F}\right]$ fluoro-L-DOPA (FDOPA) provide the basis for calculating the net influx of FDOPA to the brain relative to the metabolite-corrected plasma input $\left(K_{\text {in }}{ }^{a p p} ; \mathrm{ml} \mathrm{g}^{-1} \mathrm{~min}^{-1}\right)$ and steady-state distribution volume in brain of FDOPA $\left(V_{d} ; \mathrm{ml} \mathrm{g}^{-1}\right)$, defined below.

We used combined BOLD fMRI and FDOPA-PET to examine the relationship between regional dopamine storage and functional reactivity to affective visual cues in the amygdala and in the functionally coupled anterior cingulate cortex (ACC) in 13 healthy adult men ${ }^{9-11}$. All subjects provided informed written consent according to the declaration of Helsinki and the study was approved by the local ethics committee of the Mannheim Faculty of Medicine, University of Heidelberg. Standardized clinical assessment with the Structured Clinical Interview was performed to exclude axis I and II psychiatric disorders according to the Diagnostic and Statistical Manual of Mental Disorders, 4th edition. Trait anxiety was measured with Spielberger's State-Trait Anxiety Questionnaire (Supplementary Methods online).

We used event-related BOLD fMRI to measure amygdala reactivity during the passive viewing of standardized affectively negative and neutral visual stimuli (Supplementary Methods). The general linear model in SPM5 (Statistical Parametric Mapping, see Supplementary Methods) was used to calculate single-subject activation for the contrast of negative versus neutral stimuli. Consistent with prior studies (for example, see ref. 12), the presentation of negative versus neutral stimuli elicited significant activation in the left amygdala $(x=-21, y=-1, z=-15 ; t=3.23, P=0.036$, FWE (family-wise error) corrected for volume of interest). We next examined functional coupling between amygdala and ACC using a psycho-physiological interaction analysis ${ }^{13}$. We found that the time course of activity in left amygdala was functionally coupled with the left ACC in the context of presentation of negative compared with neutral pictures (Brodmann Area 24 (BA24); $x=-6, y=2, z=41 ; t=4.48, P<0.001$, uncorrected), with the individual magnitude of functional connectivity being negatively correlated with trait anxiety (BA24; $x=-3, y=19$, $z=24 ; t=5.59, P<0.001$, uncorrected; $R=-0.86$ ).

A standard outcome parameter for FDOPA PET imaging is the net blood-brain FDOPA clearance $\left(K_{i n}{ }^{a p p}, \mathrm{ml} \mathrm{g}^{-1} \mathrm{~min}^{-1}\right)$, which is inherently underestimated as a result of uncorrected loss of decarboxylated metabolites from brain and is only imperfectly corrected from the continuously changing proportions of FDOPA and its inert plasma metabolites ${ }^{9}$. Consequently, we used a new approach to measure $V_{d}$ $\left(\mathrm{ml} \mathrm{g}^{-1}\right)$, the steady-state distribution volume of FDOPA, which is defined as the sum of all of the volumes occupied by FDOPA in brain; that is, the plasma volume, the free FDOPA volume, and the volume occupied by $\left[{ }^{18} \mathrm{~F}\right]$ fluorodopamine formed in dopamine (and to a lesser degree in serotonin and norepinephrine) fibers and stored within vesicles ${ }^{9}$. As such, $V_{d}$ can be interpreted as an index of vesicular storage

\footnotetext{
${ }^{1}$ Department of Psychiatry and Psychotherapy, Campus Charité Mitte, Charité - University Medicine Berlin, Charitéplatz 1,10117 Berlin, Germany. ${ }^{2}$ Department of Psychiatry, University of Pittsburgh, Western Psychiatric Institute and Clinic, 3811 O'Hara Street, Pittsburgh, Pennsylvania 15213, USA. ${ }^{3}$ Department of Nuclear Medicine, Johannes Gutenberg University of Mainz, Langenbeck Strasse 1, 55131 Mainz, Germany. ${ }^{4}$ Section of Systems Neuroscience, Department of Psychiatry and Psychotherapy, Faculty of Medicine Carl Gustav Carus, Technische Universität Dresden, Würzburger Strasse 35, 01187 Dresden, Germany. ${ }^{5}$ Department of Psychiatry and Psychotherapy, RWTH Aachen University, Pauwelsstrasse 30, 52074 Aachen, Germany. ${ }^{6}$ Department of Nuclear Medicine, Ludwig Maximilian University, Campus Großhadern, Marchioninistrasse 15, 81377 Munich, Germany. ${ }^{7}$ Department of Nuclear Medicine and Research Center for Advanced Science and Technology, Tokyo University, 7-3-1, Hongo, Bunkyo-ku, Tokyo, 113-0033 Japan. ${ }^{8}$ Wellcome Trust Centre for Neuroimaging, University College London, London WC1N 3BG, UK. Correspondence should be addressed to A.H. (andreas.heinz@charite.de).
} 
a
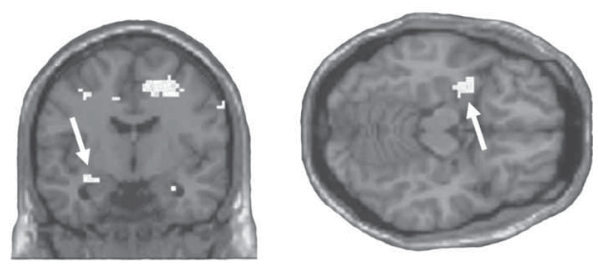

b

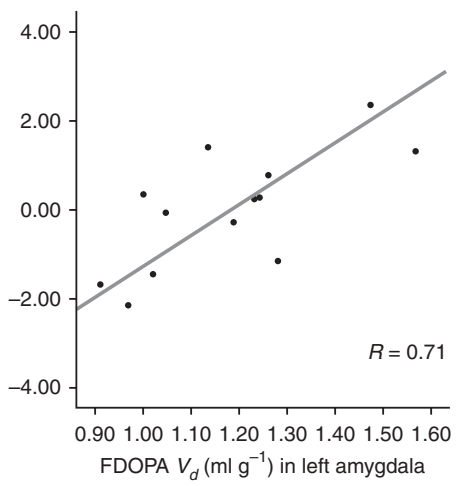

Figure 1 Correlation between FDOPA steady-state storage capacity $\left(V_{d}\right)$ in the left amygdala and fMRI BOLD response elicited by negative versus neutral stimuli in the left amygdala in 13 healthy subjects. (a) SPM5 image. The white arrow indicates the location of the left amygdala (slices at Talairach coordinates $y=-6$ and $z=-12, P=0.005$ ). (b) Correlation plot of BOLD response in the left amygdala with FOPDA $V_{d}$ in the left amygdala region of interest $(x=-30, y=-6, z=-12 ; t=3.34, P=0.035$, FWE corrected; $R=0.71$ ).

of endogenous dopamine, reflecting the 'state of readiness' for local impulse-dependent dopamine release. The magnitudes of $K_{\text {in }}{ }^{a p p}$ and $V_{d}$ were calculated in amygdala relative to the metabolite-corrected arterial FDOPA input. The mean $V_{d}$ among the 13 subjects was $1.18 \pm 0.19 \mathrm{ml}$ $\mathrm{g}^{-1}$ in the left amygdala and $1.14 \pm 0.28 \mathrm{ml} \mathrm{g}^{-1}$ in the right amygdala (Supplementary Methods, Results, Discussion and Fig. 1 online).

We regressed single-subject amygdala estimates of FDOPA $V_{d}$ against amygdala BOLD signal changes using SPM5 to characterize dopamine modulation of amygdala activity (Supplementary Methods). The magnitude of left amygdala $V_{d}$ was significantly and positively correlated with activation elicited by negative versus neutral stimuli in left amygdala $(x=-30, y=-6, z=-12 ; t=3.34, P=0.035$, FWE corrected for volume of interest; $R=0.71$; Fig. 1 ), located in close proximity to the amygdala maximum in the fMRI contrast negative versus neutral emotional stimuli reported above, and in left ACC (BA24; $x=-12, y=$ $-10, z=36 ; t=4.05, P=0.001$, uncorrected; $R=0.77$ ), located close to the ACC maximum of the connectivity analysis reported above (Supplementary Results and Discussion). The parameter $K_{i n}{ }^{a p p}$ showed no substantial correlations with functional imaging parameters. To test for specificity of these patterns, we assessed the correlation between $V_{d}$ in both amygdalae and BOLD values in the bilateral caudate, putamen and left medial frontal cortex, regions that are heavily innervated by dopamine, but were not engaged by our task; there was no significant correlation even at a liberal statistical threshold ( $P=0.01$, uncorrected). There was a weak association of $V_{d}$ in the right medial prefrontal cortex with the BOLD response in the left amygdala. However, this correlation did not reach statistical significance ( $P=0.065$; see Supplementary Results).

Our multimodal neuroimaging data highlight the importance of dopamine in regulating brain circuitry involved in processing negative emotional stimuli and mediating variability in anxious temperament. Consistent with the known potentiating effects of dopamine on amygdala function and aversive conditioning in animals ${ }^{2,3,5}$, we found that high storage of FDOPA-derived catecholamines in the left amygdala predicts activation-induced BOLD signal change in left amygdala, as well as enhanced functional coupling between left amygdala and ACC during presentation of negatively valenced visual stimuli. It should be noted that FDOPA is taken up by serotonin neurons to some extent, which also modulate amygdala responses elicited by affective stimuli ${ }^{9,12,14}$.

The functional coupling that we observed between the left amygdala and ACC was negatively correlated with trait anxiety, a finding that is consistent with studies reporting that increased functional coupling between these structures reflects a more effective integration of emotional arousal in the service of complex, adaptive behaviors and reduced negative affect $^{11}$. This pattern suggests that, although increased local dopamine release is associated with potentiated amygdala reactivity, its effect on behavior is moderated by the concurrent engagement of the ACC. Our FDOPA $V_{d}$ results suggest that the reserve of releasable dopamine in the amygdala contributes to individual differences in the responsiveness of the amygdala and functionally related prefrontal regions during processing of salient aversive stimuli. These findings support the hypothesis that dopamine encodes stimulus salience $^{1,3}$, with the amygdala being involved in the processing of aversive cues and the ventral striatum responding to errors in reward prediction. Our observations may be relevant to age-related changes in amygdala function ${ }^{15}$, given the age-dependent decrease in FDOPA $V_{d}$ in the amygdala ${ }^{14}$. It may also affect pathological emotional behaviors associated with diseases characterized by disrupted dopamine function (for example, schizophrenia and Parkinson's disease).

Note: Supplementary information is available on the Nature Neuroscience website.

\section{ACKNOWLEDGMENTS}

FDOPA plasma metabolite analysis was performed by S. Hoehnemann (University of Mainz). This study was supported by the Deutsche Forschungsgemeinschaft (HE 2597/4-3, HE 2597/7-3 and SM 80/2-2). A.R.H. is supported by the National Alliance for Research on Schizophrenia and Depression and US National Institutes of Health grant MH072837.

\section{COMPETING INTERESTS STATEMENT}

The authors declare competing financial interests: details accompany the full-text HTML version of the paper at http://www.nature.com/natureneuroscience/.

Published online at http://www.nature.com/natureneuroscience/

Reprints and permissions information is available online at http://npg.nature.com/ reprintsandpermissions/

1. Schultz, W., Dayan, P. \& Montague, P.R. Science 275, 1593-1599 (1997)

2. Pani, L., Porcella, A. \& Gessa, G.L. Mol. Psychiatry 5, 14-21 (2000).

3. Panksepp, J. Prog. Neuropsychopharmacol. Biol. Psychiatry 30, 774-784 (2006).

4. Oswald, L.M. et al. Neuroimage 36, 153-166 (2007).

5. Rosenkranz, J.A. \& Grace, A.A. Nature 417, 282-287 (2002).

6. Hariri, A.R. et al. Neuropsychopharmacology 27, 1036-1040 (2002).

7. Tessitore, A. et al. J. Neurosci. 22, 9099-9103 (2002).

8. Manuck, S.B., Brown, S.M., Forbes, E.E. \& Hariri, A.R. Am. J. Psychiatry 164, 1613-1614 (2007).

9. Kumakura, Y. et al. J. Neurosci. 27, 8080-8087 (2007).

10. Bush, G., Luu, P. \& Posner, M.I. Trends Cogn. Sci. 4, 215-222 (2000).

11. Pezawas, L. et al. Nat. Neurosci. 8, 828-834 (2005).

12. Heinz, A. et al. Nat. Neurosci. 8, 20-21 (2005).

13. Friston, K.J. et al. Neuroimage 6, 218-229 (1997).

14. Kumakura, Y. et al. Neurobiol. Aging published online, doi:10.1016/j.neurobiolaging. 2008.05.005 (9 June 2008).

15. Deeley, Q. et al. Neuroimage 40, 389-397 (2008). 